\title{
miR-375 targeting autophagy-related 2B (ATG2B) suppresses autophagy and tumorigenesis in cisplatin-resistant osteosarcoma cells
}

\author{
S. $\mathrm{GAO}^{1}, \mathrm{~K}$. WANG ${ }^{2}, \mathrm{X}$. WANG $\mathrm{WAN}^{3, *}$ \\ ${ }^{1}$ Operation Room, Linyi Cancer Hospital, Linyi, China; ${ }^{2}$ Department of Joint Surgery, Liaocheng People's Hospital, Liaocheng, China; \\ ${ }^{3}$ Department of Traumatology, Wendeng Osteopathic Hospital, Wendeng, Shandong, China \\ ${ }^{*}$ Correspondence: huayuansoho2107@163.com
}

Received April 23, 2019 / Accepted September 18, 2019

\begin{abstract}
Acquisition of drug resistance is an enormous obstacle for osteosarcoma (OS) treatment. Copious microRNAs (miRNAs) have been reported to participate in chemoresistance. However, the role and molecular basis of miR-375 in cisplatin (Cis/ DDP) sensitivity in OS remain unclear. Expression of miR-375 and autophagy-related 2B (ATG2B) in OS was examined by reverse-transcription qPCR (RT-qPCR) and western blot assay. The value of 50\% growth inhibitory concentration (IC50) for DDP was determined by Cell Counting Kit (CCK)-8 assay. Cell autophagy was measured with cell proliferation, apoptotic rate, acridine orange-positive cells, and LC3-II/LC3-I ratio determined by MTT assay, flow cytometry, and western blot assay. The candidate target of miR-375 was predicted using TargetScan and was further validated by luciferase reporter and western blot analyses. Mice xenograft model was established to further investigate the tumorigenesis of U2OS/DDP in vivo. In the study, miR-375 was downregulated in DDP-resistant OS tissues and cell lines U2OS and MG63. Elevated miR-375 or reduced ATG2B decreased cell proliferation, acridine orange-positive cells and LC3-II/LC3-I ratio, and increased apoptosis rate in U2OS/DDP and MG63/DDP cells, as accompanied with lower IC50 value for DDP. ATG2B was identified to be a target of miR-375. Re-expression of ATG2B abolished miR-375-mediated effects on cell autophagy. Moreover, the reintroduction of miR-375 slowed down tumor growth of U2OD/DDP cells in vivo. In conclusion, miR-375 suppressed cells autophagy and tumor growth in DDP-resistant U2OS/DDP and MG63/DDP cells by targeting ATG2B, providing a potential target for the treatment of DDP-resistant OS.
\end{abstract}

Key words: miR-375, DDP-resistant osteosarcoma, ATG2B, autophagy

Osteosarcoma (OS) is the most common primary malignant bone tumor and derives from primitive bone-forming mesenchymal cells, mainly occurring in children and adolescents [1]. The high incidence of OS during the adolescence growth spurt implies the possible correlation between this disease and bone development [2]. Those patients who only suffer from surgery and/or radiotherapy exhibit poor outcomes [3]. In recent years, the introduction of multiple anti-cancer drugs, including cisplatin (Cis/DDP), doxorubicin, and methotrexate dramatically prolongs the fiveyear relative survival rate of OS patients to almost 70\% [4]. However, the frequent acquisition of drug-resistant phenotypes has become the primary cause of a chemotherapy failure [5]. Thus, it is imperative to understand the molecular mechanisms underlying DDP resistance of OS and to develop novel strategies for this disease therapy.

Autophagy is a highly conserved self-catabolic degradation process, which should be responsible for the degradation of intracellular components, including proteins and damaged organelles [6, 7]. Reliable evidence has demonstrated that autophagy is essential for cell growth and survival by inducing energy production under cellular stresses conditions like hypoxia and chemotherapy [8]. Inhibition of autophagy promotes DDP-induced cell apoptosis and enhances the chemosensitivity of multiple human cancer cells to DDP, including OS [9-12].

MicroRNAs (miRNAs) are a class of small non-protein coding RNAs not longer than 18-23 nucleotides that generally modulate gene expression at the post-transcriptional level by binding to the 3' untranslated regions (3'UTR) of their target gene [13]. miRNAs can serve as tumor suppressors or oncogenes in several types of cancers by targeting genes implicated in cell proliferation, differentiation, survival, apoptosis, and metastasis $[14,15]$. In addition, mounting miRNAs have been shown to link to the chemotherapy resistance of OS to DDP $[16,17]$. miR-375 is initially identified as a pancreatic islet-specific miRNA that mainly regulates insulin secretion [18]. Following miRNA expres- 
sion profiling studies disclosed that miR-375 is frequently downregulated in a variety of human cancers, including gastric cancer [19], squamous cervical cancer [20], esophagus cancer [21], and glioma [22]. Moreover, the regulation of miR-375 in tumor sensitivity to chemotherapeutic agents has been also elucidated [23, 24]. Previous studies have revealed the anti-tumor activity of miR-375 in OS, however, the exact roles and molecular mechanisms of miR-375 in DDP resistance require to be further investigated.

In the present study, we performed a series of experiments to explore the role of miR-375 in DDP resistance in OS, as well as its underlying mechanism.

\section{Patients and methods}

Osteosarcoma tissue samples and cell lines. Fresh OS tissues were collected from 70 cases of OS patients who underwent surgical resection at the Second People's Hospital of Wuhu between March 2015 and November 2017 and affirmed by histopathological diagnosis. All patients were treated preoperatively with cisplatin-based chemotherapy rather than radiotherapy or immunotherapy. The Cis-sensitive controls consist of 35 OS patients who showed $\geq 90 \%$ tumor necrosis following the Cis treatment. The remaining 35 patients were defined as the Cis-resistant group who showed $<90 \%$ tumor necrosis. After surgery, all samples were immediately frozen in liquid nitrogen and stored at $-80^{\circ} \mathrm{C}$ until further use. This study was approved by the Research Ethics Committee of the Second People's Hospital of Wuhu and written informed consents were obtained from all participants.

Human hFOB1.19 osteoblasts, and two OS cell lines U2OS and MG63 were obtained from Cell Resource Center of Institute of Basic Medical Sciences, Chinese Academy of Medical Sciences (Beijing, China). DDP-resistant OS cell lines U2OS/DDP and MG63/DDP were developed using an intermittent stepwise selection protocol [25]. All cells were grown in Dulbecco's modified Eagle's medium (DMEM, Gibco, Carlsbad, CA, USA) in presence of $10 \%$ fetal bovine serum (FBS, Gibco) and 1\% streptomycin/ penicillin (Solarbio, Beijing, China) at $37^{\circ} \mathrm{C}$ in a humidified atmosphere containing 5\% $\mathrm{CO}_{2}$. Moreover, U2OS/DDP and MG63/DDP cells were further treated with $2 \mu \mathrm{g} / \mathrm{ml}$ DDP to maintain the resistance.

Cell transfection. miR-375 mimics (miR-375) and negative control (miR-NC), miR-375 inhibitor (anti-miR375 ) and inhibitor control (anti-miR-NC), the siRNA targeting autophagy-related 2B (ATG2B) (si-ATG2B) and relative control (si-NC) were purchased from Thermo Fisher Scientific (Waltham, MA, USA). ATG2B expression plasmid (ATG2B) was provided by Thermo Fisher Scientific by inserting the full-length sequences of ATG2B into the pcDNA3.1 vector, with an empty vector (pcDNA) as a negative control. All RNA molecules and plasmids were transfected into U2OS/DDP and MG63/DDP using Lipofectamine 2000 (Invitrogen, Carlsbad, CA, USA).
Reverse-transcription qPCR (RT-qPCR). Total RNA was extracted from homogenized tissues and cells using TRIzol $^{\circ}$ reagent (Thermo Fisher Scientific) and reverse transcribed into complementary DNA (cDNA) using M-MLV Reverse Transcriptase Kit (Thermo Fisher Scientific) or TaqMan miRNA reverse transcription kit (Thermo Fisher Scientific). Then, qPCR was carried out using SYBR Green PCR Master Mix (Thermo Fisher Scientific) and ran on the Real-time PCR Systems (Applied Biosystems, Foster City, CA, USA). The relative expression levels of miR-375 and ATG2B were normalized to U6 or $\beta$-actin using $2^{-\Delta \Delta C t}$ method. qPCR primers were listed as below: miR-375: 5 '-CTTACTATCCGTTTGTTCGTTCG-3' (forward) and 5'-TATGGTTGTTCTCGTCTCTGTGTC-3' (reverse). U6: 5'-GCTTCGGCAGCACATATACTAAAAT-3’ (forward) and $5^{\prime}$-CGCTTCACGAATTTGCGTGTCAT- $3^{\prime}$ (reverse). ATG2B: $5^{\prime}$-TGCCATCTGCTGCATTTCAAG-3' (forward) and $5{ }^{\prime}$-GTGCTGCTACCCGGGACATTA-3' (reverse). $\beta$-actin: $5^{`}$-GGCATCGTGATGGACTCCG-3` (forward) and $5{ }^{\circ}$-GCTGGAAGGTGGACAGCGA-3` (reverse).

Cell Counting Kit (CCK)-8 assay. For drug cytotoxicity analysis, transfected or non-transfected cells $\left(1 \times 10^{4}\right)$ were seeded into 96-well plates and treated with varying concentrations of cisplatin for $72 \mathrm{~h}$. Then, cells were detached using trypsin (Solarbio) and incubated with Cell Counting Kit-8 (Solarbio) for $2 \mathrm{~h}$ at $37^{\circ} \mathrm{C}$. The absorbance at $450 \mathrm{~nm}$ was detected on a microplate reader (Thermo Fisher Scientific). Cell growth inhibition rate (IR) was calculated by using the equation: $\mathrm{IR}=\left(1-\mathrm{OD}_{\text {values of control group }} / \mathrm{OD}_{\text {values of drug group }}\right) \times 100 \%$. Drug concentration that resulted in 50\% growth inhibition (IC50) was determined from the growth inhibition curve.

For cell proliferation analysis, transfected U2OS/DDP and MG63/DDP cells were seeded into 96-well plates and incubated at $37^{\circ} \mathrm{C}$ for $24 \mathrm{~h}, 48 \mathrm{~h}$, and $72 \mathrm{~h}$, respectively. Next, the absorbance at $450 \mathrm{~nm}$ was determined on a microplate reader (Thermo Fisher Scientific).

Western blot assay. Protein samples were prepared by lysing cells in cold RIPA buffer containing $1 \%$ protease inhibitor (Sigma-Aldrich, St. Louis, MO, USA). The supernatant was harvested from cell lysate by high-speed centrifugation at $12,000 \times \mathrm{g}$ for $10 \mathrm{~min}$. Afterward, equal amounts of protein denatured at $98^{\circ} \mathrm{C}$ for 5 min were subjected to sodium dodecyl sulfate polyacrylamide gel electrophoresis (SDS-PAGE) gel, and then transferred onto polyvinylidene fluoride (PVDF) membranes (Millipore, Billerica, MA, USA). After blocking with skimmed milk, the membranes were incubated with specific antibodies against LC3-I, LC3-II, ATG2B and $\beta$-actin (Abcam, Cambridge, MA, USA) overnight at $4{ }^{\circ} \mathrm{C}$. After washing, the membranes were further incubated with horseradish peroxidase (HRP)conjugated secondary antibody (Abcam) at $37^{\circ} \mathrm{C}$ for $1.5 \mathrm{~h}$. Protein signals were visualized using an enhanced chemiluminescence reagent (GE Healthcare, Giles, UK) and quantified using Image Lab software (Bio-Rad, Hercules, CA, USA). 
Flow cytometry. U2OS/DDP and MG63/DDP cells were transfected with miR-375 or si-ATG2B alone, or cotransfected with miR-375 and pcDNA or ATG12B for $48 \mathrm{~h}$. For apoptosis analysis, the cells were trypsinized, washed with cold phosphate buffer solution (PBS), and re-suspended in $100 \mu \mathrm{l}$ binding buffer to which Annexin V-FITC and propidium iodide (PI) were added. For autophagy analysis, transfected cells were treated with $10 \mathrm{mM} 3$-methyladenine, for $24 \mathrm{~h}$ and then stained with $1 \mu \mathrm{g} / \mathrm{ml}$ acridine orange for $15 \mathrm{~min}$. The cells were harvested with trypsin and washed with PBS, followed by re-suspended into $500 \mu \mathrm{l}$ flow cytometry buffer containing 1\% FBS. Apoptotic cells and autophagic cells were quantified by flow cytometry (Thermo Fisher Scientific) after the addition of $250 \mu \mathrm{l}$ binding buffer.

Luciferase assay. Partial ATG2B 3' UTR fragments containing the predicted miR-375 binding sites was amplified by PCR and inserted into psiCHECK ${ }^{\mathrm{TM}}-2$ luciferase reporter vector plasmid (Promega, Madison, WI, USA), designated as wile-type ATG2B plasmid (ATG2B WT). Mutated ATG2B 3' UTR reporter plasmid (ATG2B MUT) with the mutant binding sites of miR-375 was generated using QuikChange II XL Site-directed Mutagenesis Kit (Agilent-Stratagene, Houston, Texas, USA). Next, the luciferase construct was transfected into U2OS/DDP and MG63/DDP cells along with miR-NC, miR-375, anti-miR-NC or anti-miR-375, followed by the measurement of luciferase activity using dual luciferase reporter assay kit (Promega).

Tumorigenesis in vivo. Male BALB/c nude mice (4-6 weeks old) were purchased from Vital River Laboratory Animal Technology (Beijing, China) and kept in special pathogen-free (SPF) condition for one week. miR-NC or miR-375-expressing U2OS/DDP cells $\left(1 \times 10^{7}\right)$ were subcutaneously transplanted into the left anterior axilla of nude mice $(n=6)$. One week thereafter, mice were intraperitoneally injected with PBS containing cisplatin $(5 \mathrm{mg} / \mathrm{kg})$ once every three days, three times total. Tumor volumes were measured weekly and calculated following the formula of $\left(\right.$ width $\times$ length $\left.^{2}\right) / 2$. Five weeks after inoculation, the mice were euthanatized and xenografts were harvested and weighted. miR-375 expression and ATG2B mRNA and protein levels in xenografts were determined by RT-qPCR and western blot analyses. This study was approved by the Animal Care and Use of Committee of the Second People's Hospital of Wuhu.

Statistical analysis. All experiments were repeated three times and results were expressed as the mean \pm standard deviation (SD). Statistical analysis was performed by Student's t-test or one-way analysis of variance (ANOVA) using SPSS 20 software and statistical significance was set at $\mathrm{p}<0.05$.

\section{Results}

miR-375 expression was decreased in cisplatin-tolerant osteosarcoma. Firstly, RT-qPCR assay showed that miR-375 frequently expressed lowly in the DDP-resistant OS cohort than that in the DDP-sensitive group (Figure 1A). Next, we further confirmed the dysregulation of miR-375 in DDP-resistant OS cell lines (U2OS/DDP and MG63/DDP). CCK-8 assay showed that the IC50 values for DDP were markedly enhanced in U2OS/DDP and MG63/DDP cells compared with that in corresponding parental cell lines (U2OS and MG63) (Figures 1B, 1C), indicating the antidrug activity of U2OS/DDP and MG63/DDP cells. Following expression analysis showed that miR-375 was downregulated in OS cells relative to normal hFOB1.19 cells, especially in U2OS/DDP and MG63/DDP cells (Figure 1D). These data suggest that miR-375 may be involved in the chemoresistance of OS to cisplatin.

Elevated miR-375 inhibited cell autophagy in DDP-resistant OS cells. To investigate the effects of miR-375 on the chemosensitivity of OS, gain-of-function analysis was performed by transfecting miR-NC or miR-375 mimics into DDP-resistant OS cells. miR-375 was highly enriched in U2OS/DDP and MG63/DDP cells following miR-375 mimics transfection (Figure 2A). Functionally, a decreased IC50 value for DDP was observed in miR-375 overexpressing U2OS/DDP and MG63/DDP cells (Figure 2B), which confirmed the inhibitory effect of miR-375 on the DDP-resistant phenotype in OS. The transformation of LC3-I to LC3-II is recognized as a key process of autophagosome formation, and the ratio of LC3-II/LC3-I is commonly used as a marker of autophagy [26]. The results of the western blot assay showed that elevated expression of miR-375 resulted in the reduction of LC3-II and LC3-I ratio (Figure 2C). Acridine orange staining was used to identify autophagic cells by flow cytometry. Acridine orange-positive cells were dramatically decreased in U2OS/DDP and MG63/DDP cells when transfected with miR-375 mimic (Figures 2D, 2E). Moreover, the proliferation ability was attenuated, and apoptosis was enhanced in U2OS/DDP and MG63/DDP cells following miR-375 enrichment (Figures 2F-I), which were detected by CCK- 8 and flow cytometry analyses. In a word, miR-375 could suppress cell autophagy in U2OS/DDP and MG63/ DDP cells, as accompanied by decreased cell proliferation and promoted apoptosis rate. These results suggested a potential suppressive role of miR-375 upregulation in DDP resistance in OS cells.

ATG2B was a bona fide target of miR-375. Next, the TargetScan online website was employed to identify candidate miR-375 target genes. Among likely targets, ATG2B was picked out due to its involvement in autophagy regulation (Figure 3A). To confirm the true interaction between miR-375 and ATG2B by predicted binding sites, we constructed luciferase reporter containing wide type or mutant ATG2B 3'UTR. Luciferase assay indicated that overexpression of miR-375 inhibited, and knockdown of miR-375 promoted the luciferase activity of ATG2B WT reporter in U2OS/DDP and MG63/DDP cells but had no significant change on the luciferase activity of ATG2B MUT reporter (Figures 3B-E). Next, we further demonstrated that ATG2B was remarkably 
A

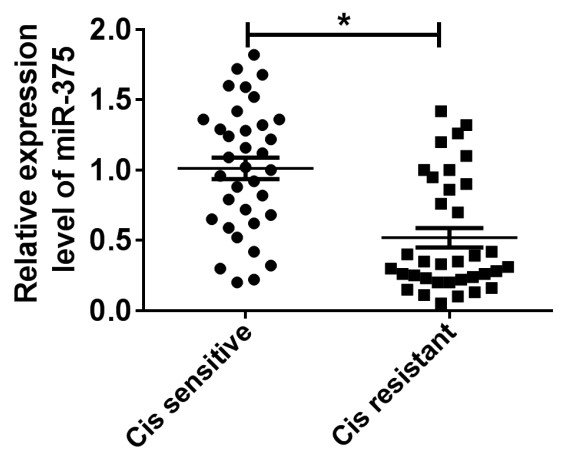

C

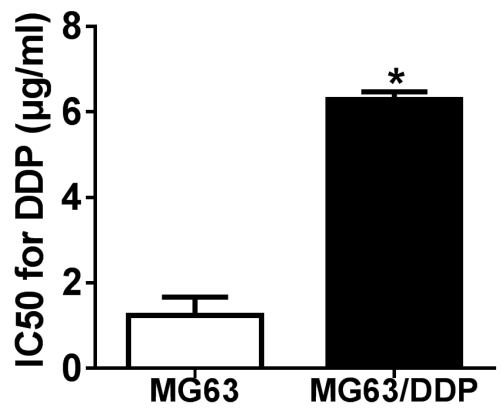

B

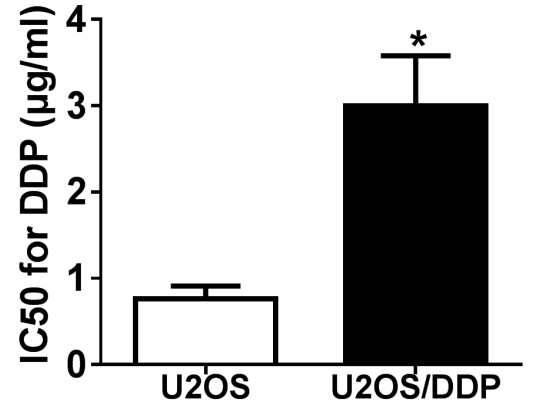

D

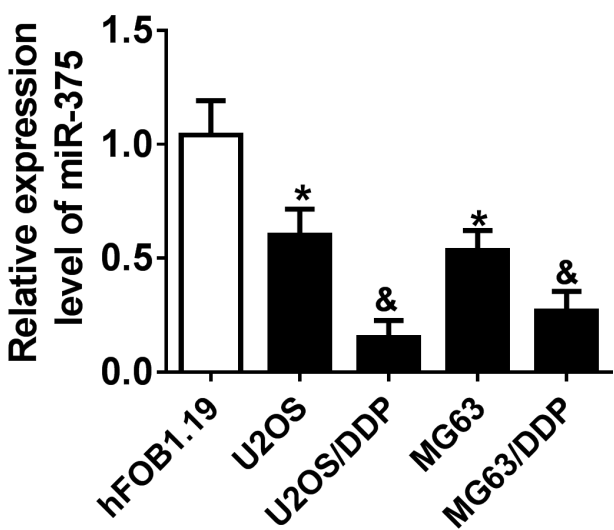

Figure 1. Attenuated expression of miR-375 in Cisplatin (Cis/DDP) resistant osteosarcoma (OS) tissues and cell lines. A) Expression of miR-375 in Cis sensitive $(n=35)$ or Cis resistant $(n=35)$ OS tissues was measured by reverse-transcription $q P C R(R T-q P C R)$. B and C) The IC50 values for DDP in parental cell lines (U2OS and MG63) and DDP-resistant cell lines (U2OS/DDP and MG63/DDP) were calculated by CCK-8 assay. D) The RT-qPCR assay was used to detect miR-375 level in hFOB1.19, U2OS, MG63, U2OS/DDP, and MG63/DDP cells. ${ }^{*}$ p $<0.05$.

upregulated in U2OS/DDP and MG63/DDP cells compared to parental counterparts (Figure 3F). Additionally, ATG2B protein expression was markedly suppressed by miR-375 overexpression, and facilitated by miR-375 inhibition in U2OS/DDP and MG63/DDP cells (Figure 3G). These results agree with the fact that miR-22 targeted 3' UTR of HMGB1 and suppressed its expression.

The knockdown of ATG2B inhibited cell autophagy in DDP-resistant OS cells. The effect of ATG2B on the drugsensitivity of OS was further investigated by the transfection of si-NC or si-ATG2B into U2OS/DDP and MG63/DDP cells. As shown in Figures $4 \mathrm{~A}$ and $4 \mathrm{~B}$, the transfection of si-ATG2B notably receded the expression of $A T G 2 B$ at mRNA and protein levels, hinting that si-ATG2B could be used for the loss-of-function analysis. Functionally, the silencing of ATG2B sensitized OS cells to DDP, which was demonstrated by the decreased IC50 value in the si-ATG2B group (Figure 4C). Moreover, ATG2B knockdown remarkably lowered LC3-II and LC3-I ratio and acridine orange-positive cells, inhibited cell proliferation and stimulated apoptosis in
U2OS/DDP and MG63/DDP cells (Figures 4D-G). These findings suggested that ATG2B downregulation was implicated in the DDP sensitivity of OS cells by reducing cell autophagy and proliferation and increasing apoptotic rate.

ATG2B abolished the suppressive effect of miR-375 on cell autophagy in OS cells. Here, we initially observed a dramatic upregulation of ATG2B both at mRNA and protein levels following transfection of ATG2B overexpressed plasmid in U2OS/DDP and MG63/DDP cells (Figures 5A, $5 \mathrm{~B})$. Following rescue experiments displayed that enforced ATG2B abolished miR-375-mediated DDP-sensitivity, displayed by the increase of IC50 value in U2OS/DDP and MG63/DDP cells (Figure 5C). Additionally, LC3-II and LC3-I ratio and acridine orange-positive cells, initially decreased by miR-375 introduction, were markedly elevated following ATG2B overexpression (Figures 5D-F). Moreover, re-expression of ATG2B counteracted the inhibitory effect of miR-375 on cell proliferation, and the promotive effect on cell apoptosis both in U2OS/DDP and MG63/DDP cells (Figures 5G-I). Altogether, these results indicated that 


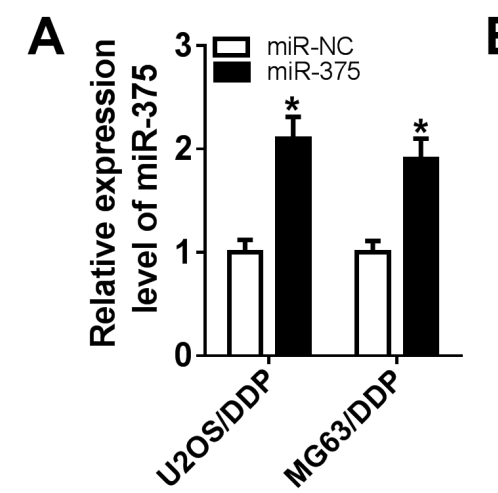

D
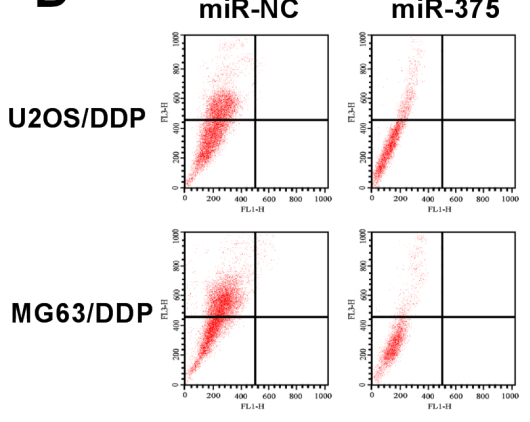

F

U2OS/DDP

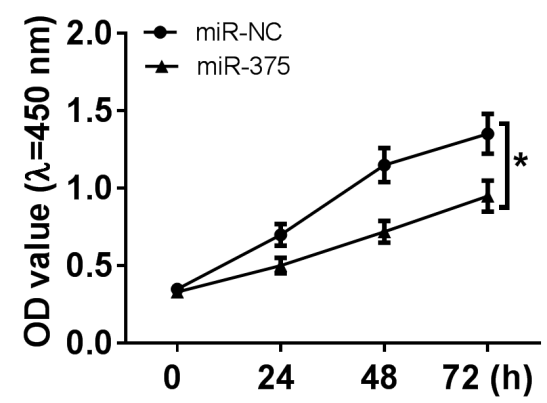

H

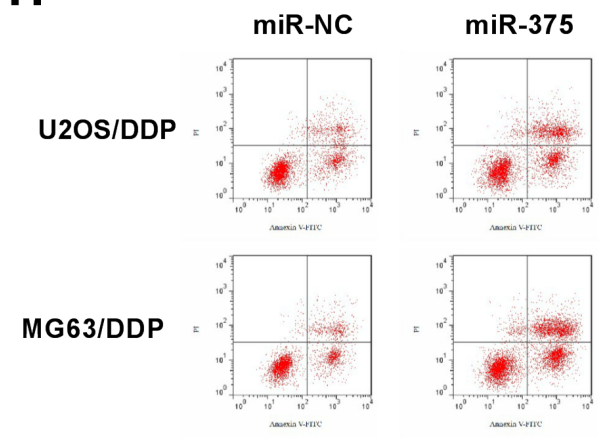

C

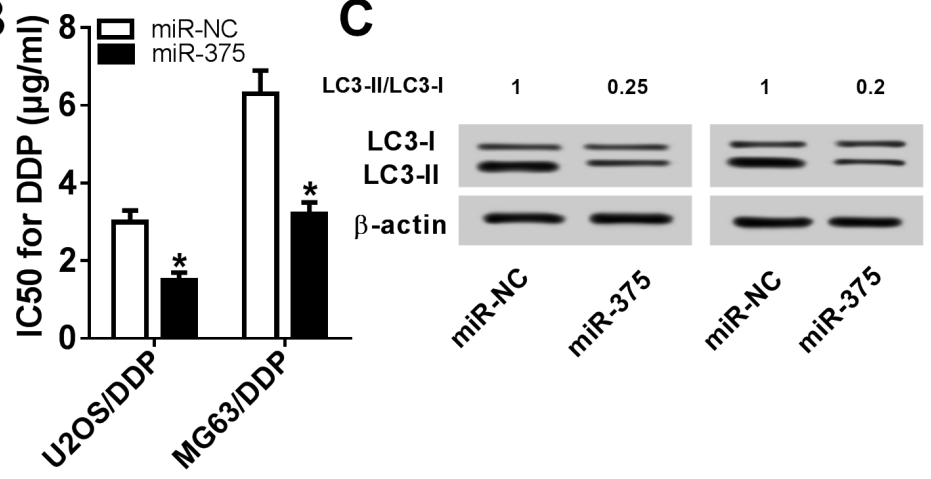

E

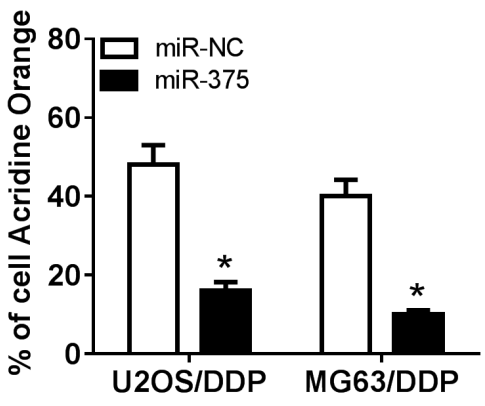

G

MG63/DDP
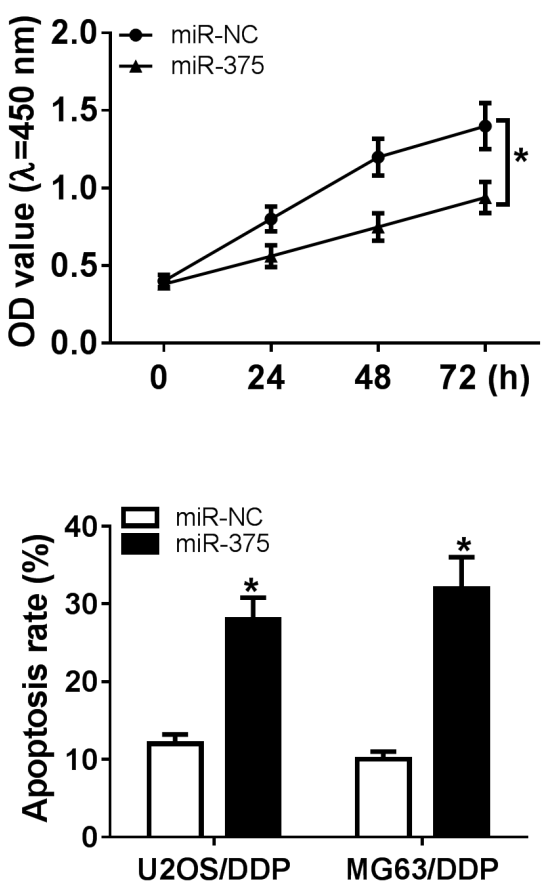

Figure 2. miR-375 modulated cell autophagy in DDP-resistant OS cells. U2OS/DDP and MG63/DDP cells were transfected with miR-NC or miR-375. A) The abundance of miR-375 was determined by RT-qPCR assay. B) The IC50 value for DDP was tested by CCK-8 assay. C) Protein expression of LC3-I and LC3-II was determined by western blot assay. D and E) Autophagic cells were identified by acridine orange staining on flow cytometry and measured using the $488 \mathrm{~nm}$ excitation detector (green fluorescence/ FL1) and the $540 \mathrm{~nm}$ emission detector (red fluorescence/ FL3) Representative FACS plots were shown and acridine orange-positive cells were calculated as $\%$ of total cells. F and G) Cell proliferation activity at different times after transfection was assessed by Cell Counting Kit (CCK)-8 assay. $\mathrm{H}$ and I) Cell apoptotic rates were detected by flow cytometry using Annexin V-FITC and PI double staining. 
A

hsa-miR-375

ATG2B WT

ATG2B MUT
3' AGUGCGCUCGgCUUGCUUGUUU 5'

5 ' ...UUGUGCUCAUCUCAGGAACAAAA... 3'

5' ...UUGUGCUCAUCUCAGCUUGUUAA... 3'
B

U2OS/DDP
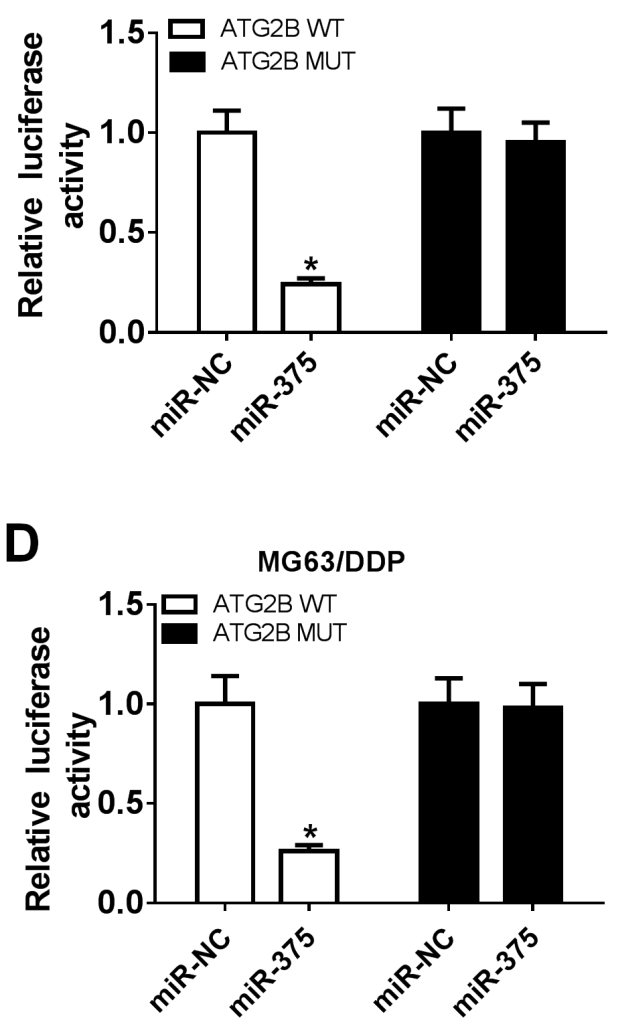

$\mathbf{F}$

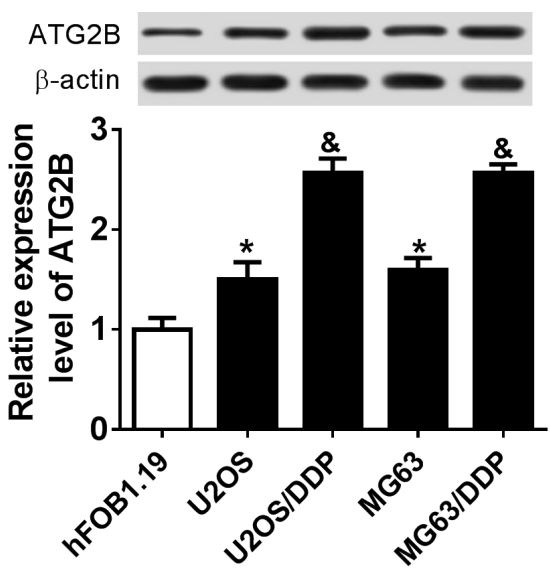

C

U2OS/DDP

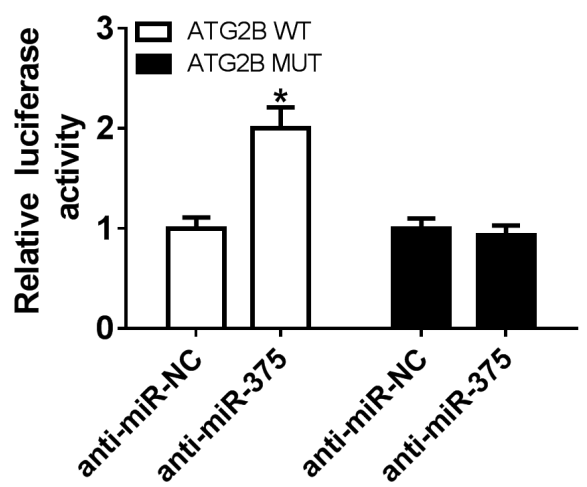

$E$

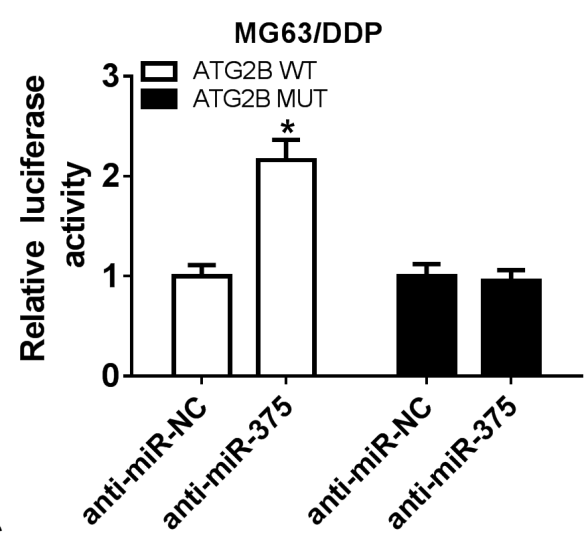

$G$
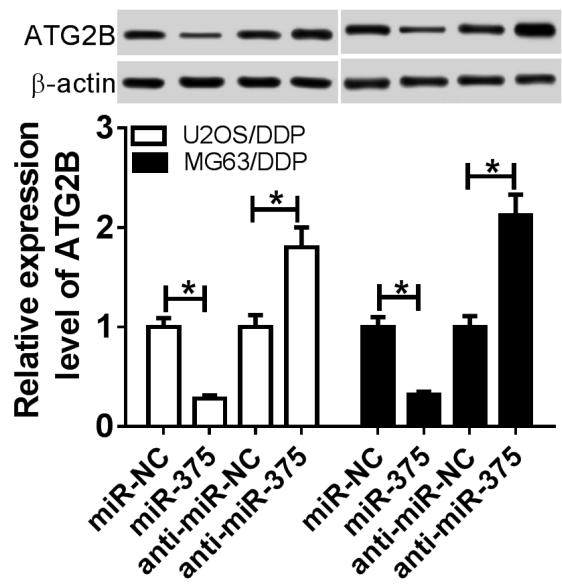

Figure 3. miR-375 directly targeted autophagy-related 2B (ATG2B) and repressed its expression. A) Putative binding sites between miR-375 and ATG2B 3'UTR were predicted by TargetScan online website, as well as the mutant sites in ATG2B MUT reporter. B-E) U2OS/DDP and MG63/DDP cells were cotransfected with ATG2B WT or ATG2B MUT reporter and miR-NC or miR-375. Then, luciferase activity was examined by luciferase reporter assay. F) ATG2B protein expression was measured by western blot assay in hFOB1.19, U2OS, MG63, U2OS/DDP, and MG63/DDP cells. G) ATG2B protein level was tested in U2OS/DDP and MG63/DDP cells following miR-375 mimics or inhibitor transfection. ${ }^{*} \mathrm{p}<0.05$. 

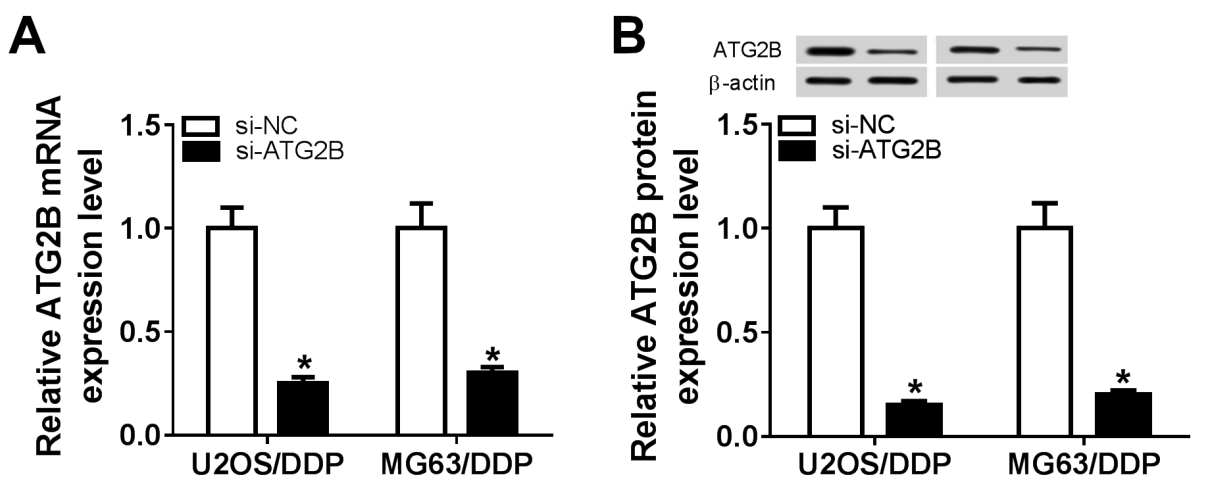

C

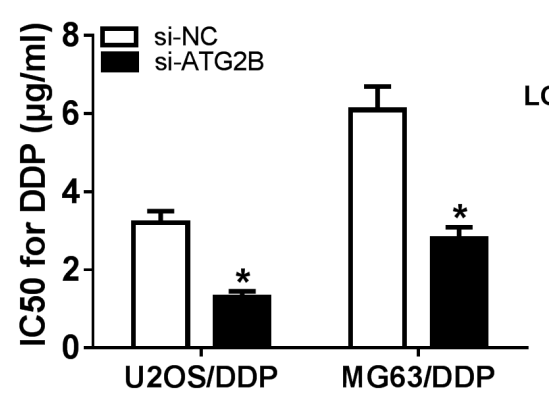

D

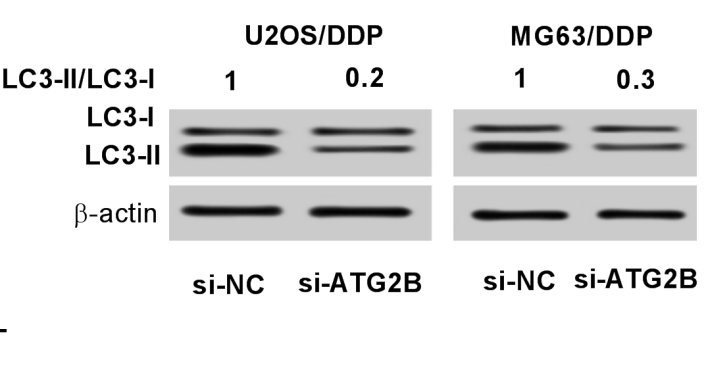

E

$\mathbf{F}$

U2OS/DDP
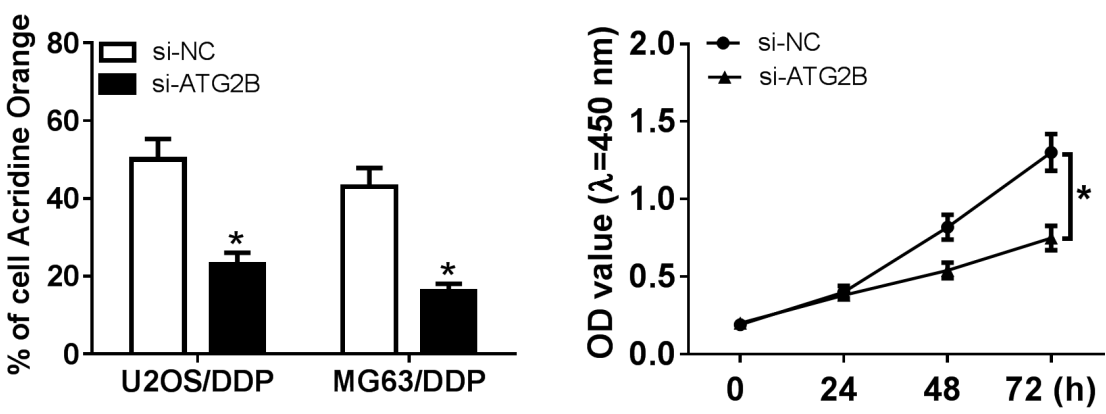

G

MG63/DDP

H
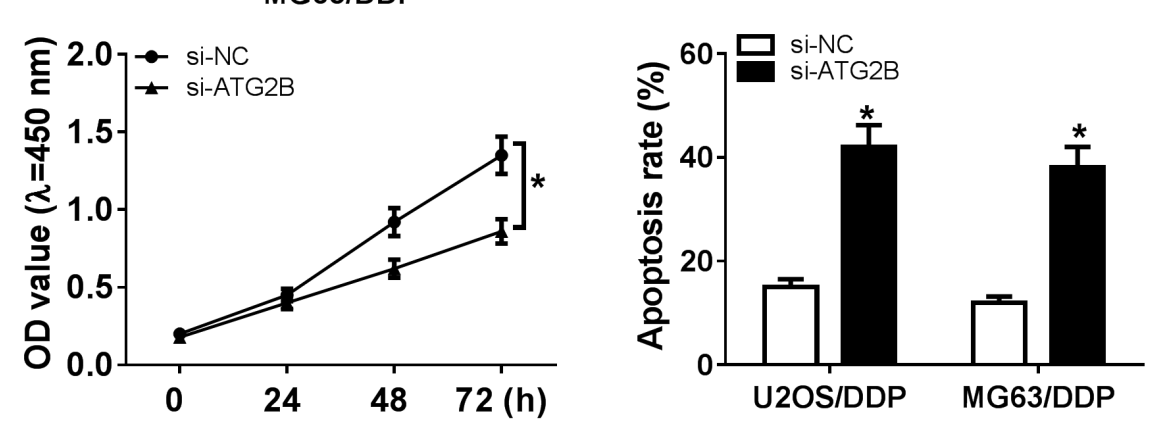

Figure 4. The knockdown of ATG2B showed a similar effect to miR-375 upregulation on cell autophagy in DDP-resistant OS cells. U2OS/DDP and MG63/DDP cells were transfected with si-NC or si-ATG2B. A and B) The expression levels of ATG2B mRNA and protein were examined by RT-qPCR and western blot assays. C) The effect of si-ATG2B on DDP sensitivity was evaluated relying on the IC50 value for DDP. D) Protein abundance of LC3-I and LC3-II was measured by western blot assay. E) Acridine orange-positive cells were calculated. F and G) CCK-8 assay was performed to assess cell proliferation activity. H) Cell apoptotic rates were detected by flow cytometry assay using Annexin V-FITC and PI double staining. 
A

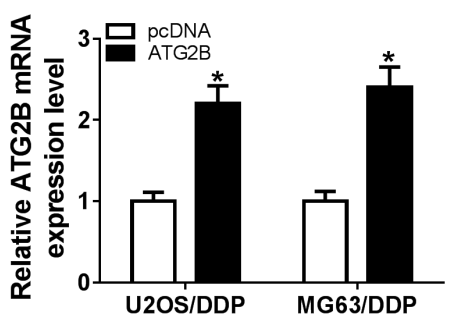

D

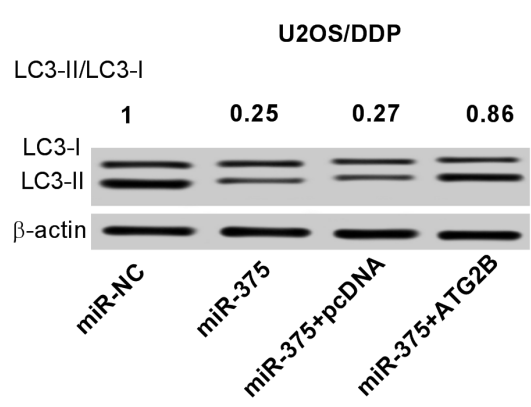

G

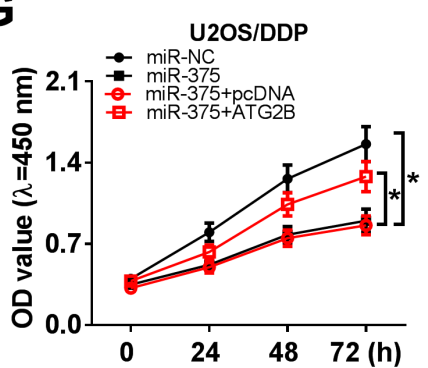

B

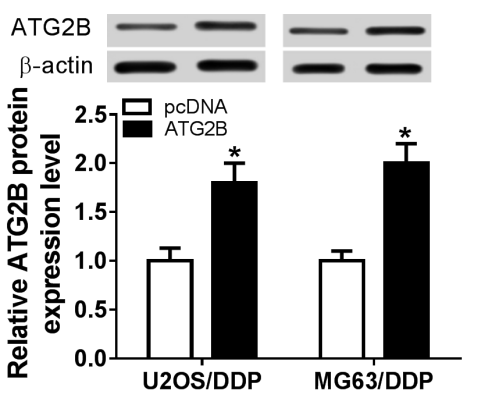

E

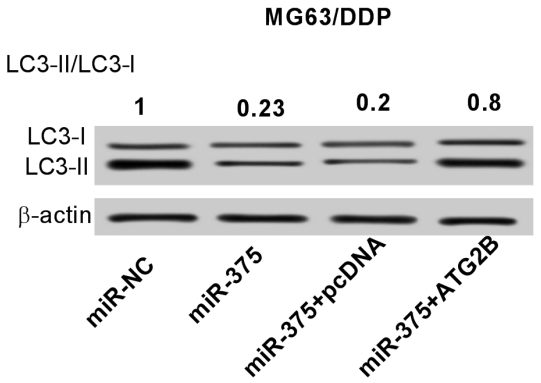

H

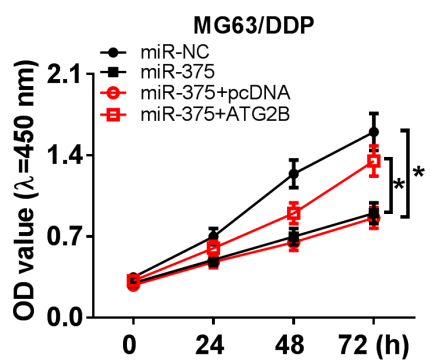

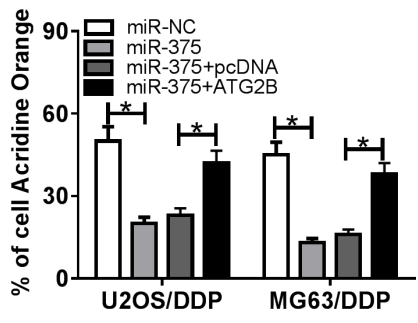

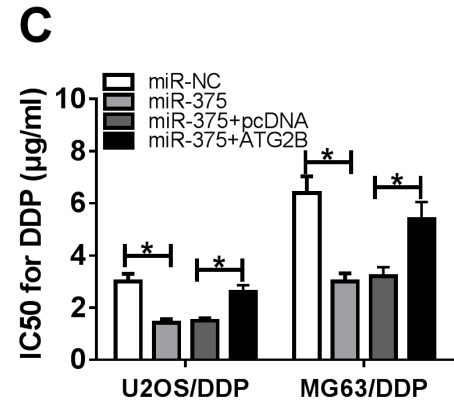

F

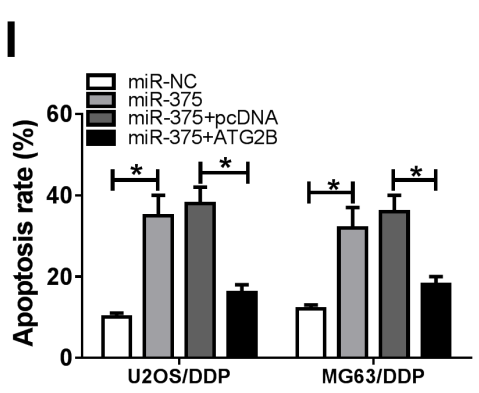

Figure 5. Restoration of ATG2B counteracted the effects of miR-375 on cell autophagy in DDP-resistant OS cells. A and B) U2OS/DDP and MG63/DDP cells were transfected with pcDNA or ATG2B. The expression levels of ATG2B mRNA and protein were detected by RT-qPCR and western blot assays. C) U2OS/DDP and MG63/DDP cells were transfected with miR-NC or miR-375 alone or cotransfected with miR-375 and pcDNA or ATG2B. At 48 h post-transfection, the IC50 value for DDP was detected by CCK-8 assay. D and E) Protein expressions of LC3-I and LC3-II were examined by western blot assay. F) Acridine orange-positive cells were calculated. G and H) Cell proliferation ability was determined by CCK-8 assay. I) Cell apoptotic rates were measured by flow cytometry using Annexin V-FITC and PI double staining.

miR-375 inhibited cell proliferation and autophagy, and suppressed the apoptotic rate in DDP-resistant OS cells by targeting ATG2B.

miR-375 served as a suppressor via repressing ATG2B in vivo. To further validate the sensitization of miR-375 in OS, nude mouse tumorigenesis assay was performed, and results showed that the introduction of miR-375 significantly blocked DDP-tolerant OS tumor growth including tumor volume and weight after cisplatin treatment (Figures 6A, $6 \mathrm{~B})$. Moreover, the introduction of miR-375 mimics lowered the level of miR-375, while enhanced the abundances of ATG2B mRNA and protein in xenografts (Figures 6C-E). Collectively, the addition of miR-375 slowed down the tumor growth of DDP-resistant OS cells in vivo.

\section{Discussion}

Chemotherapeutic resistance to multiple drugs contributes to the failure in the blockade of cancer progression. Autophagy is considered to be an intracellular self-protective mechanism that plays a chief role in degrading abnormal proteins and organelles to maintain metabolic homeostasis [27]. Activated autophagy has been found in a wide range of cancer cells in response to metabolic, hypoxia, and therapeutic stress. Moreover, a growing number of evidence highlights that autophagy has the potential to protect tumor cell from death following chemotherapy [28, 29]. As reported by Vazquez-Martin et al., knockdown of LC3 resulted in a significant reduction of cell proliferation in trastuzumab-resis- 
A

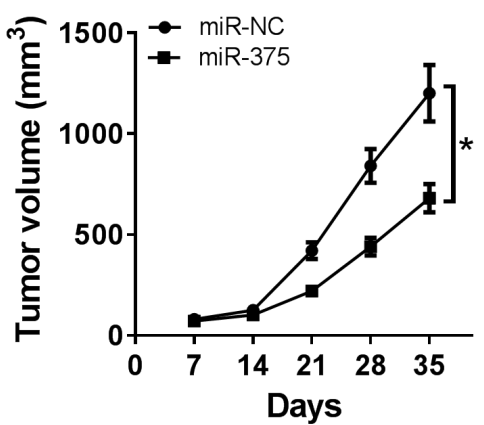

D

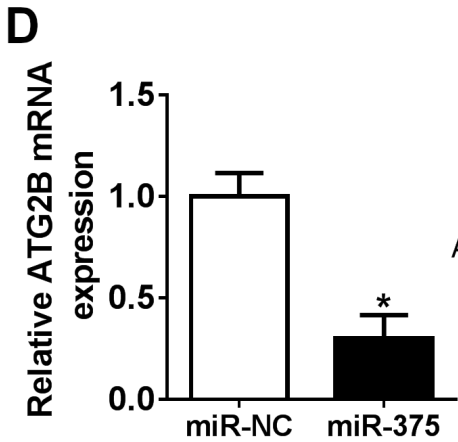

B

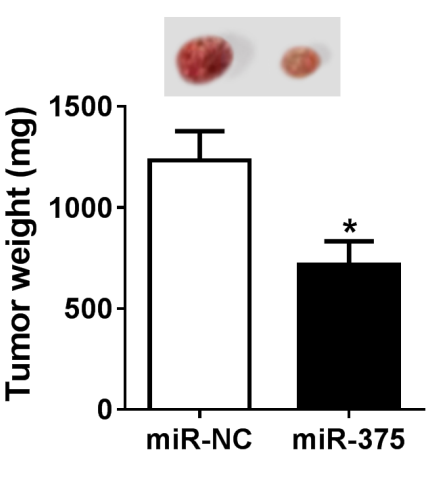

$\mathbf{E}$
C

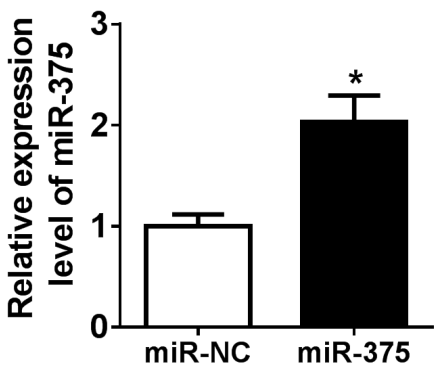

Figure 6. miR-375 slowed down tumor growth of DDP-resistant OS cells in vivo. A) Tumor volumes in the miR-NC or miR-375 group were measured weekly after inoculation and DDP treatment. B) Five weeks after inoculation, tumors were harvested and weighted. One representative image of the tumor from each group is shown. C) miR-375 expression in each group was detected by RT-qPCR analysis. D and E) The mRNA and protein levels of ATG2B in tumors were determined by RT-qPCR and western blot analyses.

tance breast cancer cells [30]. Xu et al. stated that autophagy degraded cisplatin cytotoxicity by protecting cervical cancer cells from endoplasmic reticulum stress-induced cell apoptosis [31]. Additionally, plenty of literature also revealed that blockage of autophagy augmented the sensitivity of OS cells to cisplatin [32]. Hence, therapeutic targeting autophagosome formation may become a novel approach to enhance the efficiency of chemotherapy agents in cancer.

miR-375, well known as a cancer-related miRNA, has been reported to be dysregulated in several malignant tumors and associated with cancer progression. Increasing evidence has demonstrated the dichotomous roles of miR-375 in mediating chemoresistance of various cancers. On one hand, the upregulation of miR-375 restores the sensitivity of HER2-positive breast cancer cells to trastuzumab by interacting with insulin-like growth factor 1 receptor (IGF1R) [33]. Also, miR-375 expression decreased by promoter methylation enhanced multi-drug resistance in breast cancer cells via targeting Y-box binding protein-1 (YBX1) [34]. On the other hand, elevated expression of miR-375 may contribute to epithelial-mesenchymal transition (EMT), leading to paclitaxel resistance in cervical cancer [35]. By targeting SEC23A and YAP1, miR-375 confers the chemoresistance of prostate cancer to docetaxel [36]. In the present study, we confirmed that miR-375 was notably downregulated in DDP-resistant OS tissues and cell lines, indicating the possible correlation between miR-375 and DDP resistance. Enforced expression of miR-375 suppressed cell proliferation and autophagy and promoted apoptosis rate in DDP-resistant OS cells in vitro and in vivo. Moreover, the miR-375 enrichment showed a suppressive role in DDP-resistant OS cells depending on downregulating its target gene ATG2B. These data indicated that miR-375 was associated with DDP sensitivity in OS cells partly through suppressing autophagy-mediated cell growth and promoting cell apoptosis. However, the molecular basis of miR-375 in DDP resistance requires to be further investigated.

ATG2B is an autophagy-related gene involving in the initial steps of autophagosome formation by interacting with ATG2A and WDR45 [37]. Recently, an increasing number of evidence has demonstrated that ATG2B can serve as a major modulator in cancer. For example, frameshift mutations were observed in the coding sequence of the ATG2B gene in gastric and colorectal cancer, indicating the possible correlation between ATG2B and cancer progression. miR-143b blocked tumor growth via directly targeting ATG2B in non-small cell lung cancer (NSCLC) [38]. miR-130a enhanced the killing of chronic lymphocytic leukemia cells via impeding ATG2Bmediated autophagy [39]. In addition, elevated miR-143 weakened autophagy partly via downregulating ATG2B, 
which ultimately heightened the sensitivity of prostate cancer to radiation [40]. In OS, downregulation of miR-143 accompanied by the increases of ATG2B, Bcl-2, and/or LC3-II protein levels, leading to the chemoresistance of OS tumor cells to doxorubicin [41]. Here, by luciferase reporter assay, we proved that ATG2B was a direct target of miR-375. The knockdown of ATG2B might enhance DDP sensitivity in OS cells by blockade of autophagy, which was absolutely similar to the effect of miR-357 overexpression. Furthermore, rescue experiments validated that re-expression of ATG2B abolished miR-375-mediated DDP sensitivity, cell autophagy, and growth.

In OS, miR-375 was well documented as a tumor suppressor partially by targeting downstream genes phosphatidylinositol-4, 5-bisphosphate 3-kinase, catalytic subunit alpha (PIK3CA) [42] and Yes-associated protein 1 (YAP1) [43]. The tumor-suppressive function of miR-375 was mediated by YAP1 inhibition; however, whether PIK3CA dysregulation underlay miR-375 role in OS remains undisclosed, even though PIK3CA was identified to be negatively regulated by miR-375 in a linear correlation in OS tissues and cells. In this study, we confirmed that miR-375 upregulation might be a suppressor in DDP-resistant OS cells both in vitro and in vivo by targeting ATG2B. Meanwhile, more investigations should be carried out to compare the difference of miR-375/ATG2B axis in DDP resistant cell lines and normal OS cell lines that are not resistant to DDP in further study, as well as the contribution of miR-375/message RNAs network in DDP resistance.

In conclusion, these data suggested that overexpression of miR-375 suppressed cell proliferation and autophagy, promoted apoptotic rate in DDP-resistant U2OS/DDP and MG63/DDP cells by inhibiting ATG2B, highlighting the diagnostic and therapeutic potential of miR-375 in OS patients with DDP resistance.

\section{References}

[1] OTTAVIANI G, JAFFE N. The Epidemiology of Osteosarcoma. Cancer Treat Res 2009; 152: 3-13. https://doi. org/10.1007/978-1-4419-0284-9_1

[2] HE H, NI J, HUANG J. Molecular mechanisms of chemoresistance in osteosarcoma (Review). Oncol Lett . 2014; 7: 1352-1362. https://doi.org/10.3892/ol.2014.1935

[3] MARINA N, GEBHARDT M, TEOT L, GORLICK R. Biology and therapeutic advances for pediatric osteosarcoma. Oncologist 2004; 9: 422-441. https://doi.org/10.1634/theoncologist.9-4-422

[4] SIEGEL RL, MILLER KD, JEMAL A. Cancer Statistics, 2017. CA Cancer J Clin 2017; 67: 7-30. https://doi.org/10.3322/ caac. 21387

[5] LI X, WANG S, CHEN Y, LIU G, YANG X. miR-22 targets the 3' UTR of HMGB1 and inhibits the HMGB1-associated autophagy in osteosarcoma cells during chemotherapy. Tumour Biol 2014; 35: 6021-6028. https://doi.org/10.1007/ s13277-014-1797-0
[6] SU Z, YANG Z, XU Y, CHEN Y, YU Q. MicroRNAs in apoptosis, autophagy and necroptosis. Oncotarget 2015; 6: 84748490. https://doi.org/10.18632/oncotarget.3523

[7] LEVINE B, KROEMER G. Autophagy in the Pathogenesis of Disease. Cell 2008; 132: 27-42. https://doi.org/10.1016/j. cell.2007.12.018

[8] MIZUSHIMA N, LEVINE B, CUERVO AM, KLIONSKY DJ. Autophagy fights disease through cellular self-digestion. Nature 2008; 451: 1069-1075. https://doi.org/10.1038/nature06639

[9] WANG J, WU GS. Role of autophagy in cisplatin resistance in ovarian cancer cells. J Biol Chem 2014; 289: 17163-17173. https://doi.org/10.1074/jbc.M114.558288

[10] XU Y, YU H, QIN H, KANG J, YU C et al. Inhibition of autophagy enhances cisplatin cytotoxicity through endoplasmic reticulum stress in human cervical cancer cells. Cancer Lett 2012; 314: 232-243. https://doi.org/10.1016/j. canlet.2011.09.034

[11] YU L, GU C, ZHONG D, SHI L, KONG Y et al. Induction of autophagy counteracts the anticancer effect of cisplatin in human esophageal cancer cells with acquired drug resistance. Cancer Lett 2014; 355: 34-45. https://doi.org/10.1016/j.canlet.2014.09.020

[12] LI Y, JIANG W, HU Y, DA Z, ZENG C et al. MicroRNA199a-5p inhibits cisplatin-induced drug resistance via inhibition of autophagy in osteosarcoma cells. Oncol Lett 2016; 12: 4203-4208. https://doi.org/10.3892/ol.2016.5172

[13] SINGH R, MO YY. Cancer Biol Ther 2013; 14: 201-212. https://doi.org/10.4161/cbt.23296

[14] CALIN GA, CROCE CM. MicroRNA-cancer connection: the beginning of a new tale. Cancer Res 2006; 66: 7390-7394. https://doi.org/10.1158/0008-5472.CAN-06-0800

[15] LIN S, GREGORY RI. MicroRNA biogenesis pathways in cancer. Nat Rev Cancer 2015; 15: 321-333. https://doi. org/10.1038/nrc3932

[16] ZHAO G, CAI C, YANG T, QIU X, LIAO B et al. MicroRNA-221 Induces Cell Survival and Cisplatin Resistance through PI3K/Akt Pathway in Human Osteosarcoma PLoS One 2013; 8: e53906. https://doi.org/10.1371/journal. pone.0053906

[17] GENG S, GU L, JU F, ZHANG H, WANG Y et al. MicroRNA-224 promotes the sensitivity of osteosarcoma cells to cisplatin by targeting Rac1. J Cell Mol Med 2016; 20: 16111619. https://doi.org/10.1111/jcmm. 12852

[18] POY MN, ELIASSON L, KRUTZFELDT J, KUWAJIMA S, $\mathrm{MA} X$ et al. A pancreatic islet-specific microRNA regulates insulin secretion. Nature 2004; 432: 226-230. https://doi. org/10.1038/nature03076

[19] DING L, XU Y, ZHANG W, DENG Y, SI M et al. MiR-375 frequently downregulated in gastric cancer inhibits cell proliferation by targeting JAK2. Cell Res 2010; 20: 784-793. https://doi.org/10.1038/cr.2010.79

[20] WANG F, LI Y, ZHOU J, XU J, PENG C et al. miR-375 is down-regulated in squamous cervical cancer and inhibits cell migration and invasion via targeting transcription factor SP1. Am J Pathol 2011; 179: 2580-2588. https://doi. org/10.1016/j.ajpath.2011.07.037 
[21] FU C, DONG W, WANG Z, LI H, QIN Q et al. The expression of miR-21 and miR-375 predict prognosis of esophageal cancer. Biochem Biophys Res Commun 2014; 446: 1197-203. https://doi.org/10.1016/j.bbrc.2014.03.087

[22] CHANG C, SHI H, WANG C, WANG J, GENG $\mathrm{N}$ et al. Correlation of microRNA-375 downregulation with unfavorable clinical outcome of patients with glioma. Neurosci Lett 2012; 531: 204-208. https://doi.org/10.1016/j.neulet.2012.10.021

[23] ZHOU N, QU Y, XU C, TANG Y. Upregulation of microRNA-375 increases the cisplatin-sensitivity of human gastric cancer cells by regulating ERBB2. Exp Ther Med; 11: 625630. https://doi.org/10.3892/etm.2015.2920

[24] YANG D, YAN R, ZHANG X, ZHU Z, WANG C et al. Deregulation of MicroRNA-375 inhibits cancer proliferation migration and chemosensitivity in pancreatic cancer through the association of HOXB3. Am J Transl Res 2016; 8: 1551-1559.

[25] MIURA N, TAKEMORI N, KIKUGAWA T, TANJI N, HIGASHIYAMA S et al. Adseverin: A novel cisplatin-resistant marker in the human bladder cancer cell line HT1376 identified by quantitative proteomic analysis. Mol Oncol 2012; 6: 311-322. https://doi.org/10.1016/j.molonc.2011.12.002

[26] VON HOVEN G, KLOFT N, NEUKIRCH C, EBINGER S, BOBKIEWICZ W et al. Modulation of translation and induction of autophagy by bacterial exoproducts. Med Microbiol Immunol 2012; 201: 409-418. https://doi.org/10.1007/ s00430-012-0271-0

[27] LEVINE B, KLIONSKY DJ. Development by Self-Digestion: Molecular Mechanisms and Biological Functions of Autophagy. Dev Cell 2004; 6: 463-477. https://doi.org/10.1016/ s1534-5807(04)00099-1

[28] CHEN N, KARANTZA-WADSWORTH V. Role and regulation of autophagy in cancer. Biochim Biophys Acta 2009; 1793: 1516-1523. https://doi.org/10.1016/j.bbamcr.2008

[29] CHEN S, REHMAN SK, ZHANG W, WEN A, YAO L et al. Autophagy is a therapeutic target in anticancer drug resistance. Biochim Biophys Acta 2010; 1806: 220-229. https:// doi.org/10.1016/j.bbcan.2010.07.003

[30] VAZQUEZ-MARTIN A, OLIVERAS-FERRAROS C, MENENDEZ JA. Autophagy facilitates the development of breast cancer resistance to the anti-HER2 monoclonal antibody trastuzumab. PLoS One 2009; 4: e6251. https://doi. org/10.1371/journal.pone.0006251

[31] XU Y, YU H, QIN H, KANG J, YU C et al. Inhibition of autophagy enhances cisplatin cytotoxicity through endoplasmic reticulum stress in human cervical cancer cells. Cancer Lett 2012; 314: 232-243. https://doi.org/10.1016/j. canlet.2011.09.034
[32] ZHAO Z, TAO L, SHEN C, LIU B, YANG Z et al. Silencing of Barkor/ATG14 sensitizes osteosarcoma cells to cisplatin?induced apoptosis. Int J Mol Med 2014; 33: 271 276. https://doi.org/10.3892/ijmm.2013.1578

[33] YEXM, ZHU HY, BAI WD, WANG T, WANG L et al. Epigenetic silencing of miR-375 induces trastuzumab resistance in HER2-positive breast cancer by targeting IGF1R. BMC Cancer 2014; 14: 134. https://doi.org/10.1186/1471-2407-14-134

[34] LIU SL, SUI YF, LIN MZ. MiR-375 is epigenetically downregulated due to promoter methylation and modulates multidrug resistance in breast cancer cells via targeting YBX1. Eur Rev Med Pharmacol Sci 2016; 20: 3223-3229.

[35] SHEN Y, ZHOU J, LI Y, YE F, WAN X et al. miR-375 mediated acquired chemo-resistance in cervical cancer by facilitating EMT. PLoS One 2014; 9: e109299. https://doi. org/10.1371/journal.pone.0109299

[36] WANG Y, LIEBERMAN R, PAN J, ZHANG Q, DU M et al. miR-375 induces docetaxel resistance in prostate cancer by targeting SEC23A and YAP1. Mol Cancer 2016; 15: 70. https://doi.org/10.1186/s12943-016-0556-9

[37] BEHRENDS C, SOWA ME, GYGI SP, HARPER JW. Network organization of the human autophagy system. Nature 2010; 466: 68-76. https://doi.org/10.1038/nature09204

[38] WEI J, MA Z, LI Y, ZHAO B, WANG D et al. miR-143 inhibits cell proliferation by targeting autophagy-related $2 \mathrm{~B}$ in non-small cell lung cancer H1299 cells. Mol Med Rep 2015; 11: 571-576. https://doi.org/10.3892/mmr.2014.2675

[39] KOVALEVA V, MORA R, PARK YJ, PLASS C, CHIRAMEL AI et al. miRNA-130a targets ATG2B and DICER1 to inhibit autophagy and trigger killing of chronic lymphocytic leukemia cells. Cancer Res 2012; 72: 1763-1772. https://doi. org/10.1158/0008-5472.CAN-11-3671

[40] LIU J, LI M, WANG Y, LUO J. Curcumin sensitizes prostate cancer cells to radiation partly via epigenetic activation of miR-143 and miR-143 mediated autophagy inhibition. J Drug Target 2017; 25: 645-652. https://doi.org/10.1080/1061 186X.2017.1315686

[41] ZHOU J, WU S, CHEN Y, ZHAO J, ZHANG K et al. microRNA-143 is associated with the survival of ALDH1+CD133+ osteosarcoma cells and the chemoresistance of osteosarcoma. Exp Biol Med (Maywood) 2015; 240: 867-875. https:// doi.org/10.1177/1535370214563893

[42] LIU G, HUANG K, JIE Z, WU Y, CHEN J et al. Circfat1 sponges mir-375 to promote the expression of yes-associated protein 1 in osteosarcoma cells. Mol Cancer 2018; 17: 170. https://doi.org/10.1186/s12943-018-0917-7

[43] SHI ZC, CHU XR, WU YG, WU JH, LU CW et al. Microrna-375 functions as a tumor suppressor in osteosarcoma by targeting pik3ca. Tumour Biol 2015; 36: 8579-8584. https:// doi.org/10.1007/s13277-015-3614-9 